Anuario Latinoamericano Ciencias Políticas y Relaciones Internacionales vol. 7, 2019

pp. $329-350$

\section{La geopolítica de la corrupción en México}

\section{The Geopolitics of Corruption in Mexico}

\author{
Nubia Nieto* \\ INVESTIGADORA INDEPENDIENTE \\ $\triangle$ continents.sky@gmail.com \\ https://orcid.org/0000-0001-9454-5922
}

\section{RESUMEN}

Los mayores problemas que enfrenta la humanidad alcanzan magnitudes globales: terrorismo, cambio climático, narcotráfico y corrupción, entre otros. En este contexto, el estudio de la corrupción se hace más complejo toda vez que se funde con otros fenómenos internacionales.

La interrogante que guía el presente texto es ¿Por qué la corrupción representa un riesgo geopolítico global? La hipótesis de este trabajo supone a la corrupción como un riesgo geopolítico que amenaza a gobiernos y países de todo el mundo. Se toma como estudio de caso a México, debido a que los niveles de corrupción que experimenta este país han contribuido a acelerar otros fenómenos globales tales como el narcotráfico y otros crímenes. El objetivo principal es ofrecer una radiografía de la nueva geopolítica de la corrupción mundial y de México en particular. El trabajo también presenta la perspectiva anticorrupción del gobierno mexicano de Andrés Manuel López Obrador (2018-proyectado al 2024).

PALABRAS CLAVE: geopolítica, corrupción, México, relaciones internacionales.

\section{ABSTRACT}

The biggest problems facing humanity reach global magnitudes: terrorism, climate change, drug trafficking and corruption, among others. In this context, the study of corruption becomes more complex as it merges with other international phenomena. The question that guides this text is: Why does corruption represent a global geopolitical risk? The hypothesis of this work considers to corruption as a geopolitical risk that threatens governments and countries around the world. Mexico is taken

* Doctora en Geopolítica por la Universidad de la Sorbona Panteón-París I, París, Francia. Maestría en Sociedades Latinoamericanas (DEA) por el Instituto de Altos Estudios de América Latina (IHEAL), París III. Maestría en Ciencias Políticas en la Facultad de Ciencias Políticas y Sociales de la UNAM, México. Diplomada en Estudios Latinoamericanos (DELA), por IHEAL. Diplomada en Análisis de Coyuntura Política, UNAM. Licenciaturas en Sociología y en Ciencias de la Comunicación por la Universidad Metropolitana UAM-Xochimilco, México. 
as a case study, due to the levels of corruption experienced by this country, which have contributed to accelerating other global phenomena such as drug trafficking and other crimes. The main objective is to offer a picture of new geopolitics of global corruption and of Mexico in particular. The work also presents the anti-corruption perspective of the Mexican government of Andrés Manuel López Obrador (2018 projected to 2024).

KEYWORDS: geopolitics, corruption, Mexico, international relations.

\section{Introducción}

El estudio de los fenómenos sociales es cada vez más complejo. Diferentes riesgos geopolíticos aparecen y cada vez más difícil de estudiar de manera separada, los cuales amenazan a la humanidad y al desarrollo de las democracias representativas: desigualdad social, pobreza, destrucción del medio ambiente, terrorismo, ciberseguridad, movimientos de extrema derecha, esclavismo sexual y laboral, narcotráfico, lavado de dinero y corrupción entre los más representativos. Estudiar cada uno daría material didáctico para elaborar enciclopedias de terror. No obstante, el presente texto busca desde una perspectiva geopolítica crítica describir el análisis de la corrupción global, pero centrándose en México, como un estudio de caso.

El análisis gira en torno a las siguientes cuestiones: 1) ¿Por qué la corrupción representa un riesgo geopolítico global contemporáneo?, 2) ¿cuáles son las características de la corrupción en México?, 3) ¿qué factores han provocado que la corrupción en México amenace la estructura geopolítica del Estado?, 4) ¿cuáles son las propuestas para reducir los niveles de corrupción en México?

Las hipótesis tentativas que buscan responder las anteriores preguntas son las siguientes: 1) La corrupción se ha convertido en un fenómeno global que pone en riesgo a la estabilidad de los gobiernos. 2) Las características de la corrupción mexicana deposan fronteras nacionales y adquieren dimensiones mundiales. 3) La corrupción en México fusionada con otros fenómenos globales como el crimen organizado amenazan la estructura geopolítica del Estado. 4) El gobierno de Andrés Manuel López Obrador coloca el tema de la lucha contra la corrupción como eje de legitimidad su gobierno.

Asimismo, el presente artículo se plantea los siguientes objetivos: 1) Exponer los riesgos que representa la corrupción para la geopolítica global actual. 2) Describir las características de la corrupción mexicana. 3) Analizar los elementos que han propiciado que la corrupción amenace la estructura geopolítica del Estado Mexicano. 4) Presentar brevemente las iniciativas anticorrupción planteadas por el gobierno mexicano.

El texto se divide en cuatro apartados: el primero describe los fundamentos teóricos de la geopolítica, el segundo presenta a la corrupción como un riesgo para la estabilidad de la geopolítica global, el tercero expone a la 
corrupción mexicana como un riesgo geopolítico para el Estado Mexicano, y el cuarto resume brevemente la propuesta anticorrupción del gobierno de Andrés Manuel López Obrador (AMLO). Finalmente, se presentan algunos comentarios generales.

Cabe mencionar que el artículo sigue una metodología de análisis descriptivo tomando en consideración datos cualitativos y cuantitativos, así como literatura académica, publicaciones de revistas, periódicos y reportes globales. El texto confronta datos estadísticos, entrevistas, reportajes y artículos de publicaciones tanto académicas como de investigación periodística, y por supuesto técnicas observacionales y visitas al país.

\section{Fundamentos teóricos de geopolítica}

El término geopolítica es usado habitualmente en los medios de comunicación masiva, reportes militares, y redes sociales para referirse a fenómenos globales. La palabra geopolítica según el diccionario de la Real Academia de la Lengua Española viene del griego $\gamma \tilde{\eta} g \hat{e}$-Geo- que significa tierra o territorio, y politiké-política-, la fusión de ambas palabras da lugar al estudio relacionado con el punto de vista geográfico y político de una región (RAE 2015).

En tiempos modernos, el término geopolítica fue acuñado en 1916 por el jurista sueco Rudolf Kjellen (1846-1922). Según él, la geopolítica es "el estudio del Estado como un organismo geográfico, como un fenómeno espacial, es decir un lugar delimitado por un territorio, espacio, o más exactamente por las delimitaciones territoriales de un país" (Kjellen 1916: 29). Sin embargo, esta disciplina se remonta al siglo quinto antes de Cristo, en la obra del escritor y geógrafo, Heródoto (-485/-425 A.C.). El término fue usado en la narrativa Historias, con la connotación de "averiguar" para explicar las causas y los métodos de acción en la guerra Greco-Persa (499-479 A.C). En esta obra, Heródoto va a hacer la distinción entre el mito y sucesos reales de la historia. En la construcción de los sucesos históricos, Heródoto involucra la definición de unidades geográficas tales como "Asia" y "Europa" que conecta íntimamente a la política, como una serie de interacciones hostiles entre los habitantes de las tierras persas, la conciencia de sus habitantes, y la noción de distinción geográfica. Así, Heródoto utiliza el término para explicar las causas y métodos de acción en la guerra Greco-Persa, haciendo referencia a la influencia geográfica de cada Estado persa conquistado en la configuración de la conciencia de la gente, de las costumbres y de la organización política de esos pueblos (Rood 2010: 45). Durante la Primera Guerra Mundial (1914-1918), la geopolítica fue ampliamente utilizada en el estudio de las ciencias sociales, pero después de la Segunda Guerra Mundial (1939-1945) el uso de la geopolítica se lo vinculó con el nazismo alemán en la medida que fue utilizada para justificar la invasión y control sobre otros países. La geopolítica también fue usada por el fascismo italiano y japonés, y asociada a políticas de genocidio, racismo, expansionismo y dominación territorial (Lacoste 1993: 4).
La geopolítica

de la corrupción en México

Nubia Nieto 
Sin embargo, el término geopolítica reapareció con el comienzo del conflicto entre Camboya y Vietnam a fines de los años setenta. A partir de ese momento la palabra geopolítica cobró vida en los medios de información para referirse a los conflictos territoriales, más que ideológicos. El término geopolítica se reafirmó con más fuerza durante el conflicto de Irán-Irak (1980-1988) y la invasión de Kuwait (1990-1991) en el golfo Pérsico. Sin embargo, la geopolítica cobra presencia a partir de 1989 con la caída del muro de Berlín para describir reacomodos territoriales, políticos, ideológicos y religiosos (Lorot, Thual 2002: 43).

La escuela francesa fue pionera en revitalizar a la geopolítica como una disciplina académica. Jacques Soppelsa, exdirector de la Universidad de la Sorbona y Presidente de la Academia Internacional de Geopolítica en Francia, definió a la geopolítica como la continuación de la política por otros medios: "La geopolítica, a diferencia de la geografía política - que describe la organización del mundo dividido en Estados y naciones-, tiende a explicar fenómenos fundados en la división del espacio, estructuras y configuraciones territoriales, y de elementos variables como los recursos naturales, poblaciones y estructuras político-sociales (...) La geopolítica de la época contemporánea es más que nunca la continuación de la política por otros medios" (Soppelsa et al. 1988: 129). En tanto, Yves Lacoste, autor francés del diccionario de geopolítica, considera a esta disciplina como el estudio de las rivalidades territoriales entre diferentes tipos de poder: "La geopolítica trata de las rivalidades de poder sobre los territorios" (Lacoste 1993: 587). Para Lacoste (1993) la comprensión de rivalidades territoriales de poder pasa por la intermediación de un cierto número de representaciones que se definen como el conjunto de ideas y percepciones colectivas de orden político, religioso y demás que estructuran a los grupos sociales y su percepción del mundo (Lacoste 1993: 4). Lacoste (1993) introduce nuevos elementos para el análisis de la geopolítica que no son motivados únicamente por rivalidades territoriales, sino por lógicas de representación en las que cada grupo social o individuo es producto de una educación y caracterizado por representaciones específicas, de acuerdo con su etnicidad o ubicación específica. Lacoste retoma el concepto de la producción del espacio acuñado por Henry Lefèvre (2000) para argumentar el concepto de representación en la geopolítica. Lacoste (1993) considera que entre las representaciones más comunes que configuran la geopolítica están la consciencia política, nacional, étnica, religiosa, y cargas morales y emocionales de los individuos y de las colectividades. Lacoste (1993) también introduce en el análisis de la geopolítica comparaciones históricas de periodos cortos y prolongados para ver y medir los impactos geopolíticos, y el análisis de mapeo a multi-escala, es decir, representaciones gráficas a través de la cartografía.

En tanto, Dodds (2014: 13) define a la geopolítica como una perspectiva mundial que engloba relaciones de poder, geografía y conocimiento: "El término geopolítica describe no solo territorios, sino también las representaciones y prácticas que entran en el dominio público, y ayudan al mismo 
tiempo a configurar el entendimiento de las élites encarnadas en los líderes políticos, dueños de corporaciones multinacionales, (...) y el funcionamiento de agentes sociales que se constituye desde las minorías étnicas, grupos indígenas y el conjunto de individuos que forman las sociedades" (Dodds 2014: 13). Dodds recupera el carácter dinámico del concepto de geopolítica, alejándolo de percepciones estáticas que limitan al término al estudio territorial definido por Estados, grandes poderes, y agentes de poder como los políticos. Dodds destaca la dimensión de los actores sociales o agentes humanos en la configuración del poder, del territorio y del conocimiento de ambas esferas (Dodds 2014: 17).

A su vez, Nayef Al-Rodham (2009) combina el enfoque tradicional basado en estudios territoriales, pero también agrega nuevas perspectivas multidimensionales de poder y las relaciones de poder, que llama meta-geopolítica, en el marco del uso de herramientas basadas en el soft power y hard power -poder blando y poder duro- ${ }^{1}$ que el Estado emplea para obtener y mantener el poder. Nayef Al-Rodham define siete dimensiones del poder estatal en sus categorías del análisis geopolítico: sector social, sector de la salud pública, política doméstica, economía, medio ambiente, ciencias y potencial humano. A través de estas categorías, la meta-geopolítica permite analizar las fortalezas y debilidades de los Estados, de las empresas privadas y de las empresas transnacionales que cada vez cobran más fuerza en el destino geopolítico de los países (Al-Rodham 2009).

Las contribuciones de la escuela alemana, francesa y británica han tenido importantes repercusiones en el rescate del análisis de la geopolítica como el area interdisciplinaria que recupera aspectos basados en limitaciones territoriales y fronterizas, y dimensiones fundadas en relaciones y representaciones del poder, en la generación del conocimiento técnico y científico, en las comparaciones históricas, en las narrativas culturales, sociales, en el desarrollo económico y, por supuesto, en la proyección del poder en sus formas de poder blando y poder duro. Sin dejar de lado a la representación cartográfica, estadística y gráfica del poder, y la proyección y aceptación del poder tanto al interior de los países como al exterior de estos. Desde luego, existen otras contribuciones importantes de la escuela clásica de la geopolítica representada en la teoría del poder naval del almirante estadounidense Alfred Mahan (2013), quien sostiene la importancia geoestratégica de contar con una marina de guerra poderosa. La teoría del corazón continental o heartland del geógrafo británico Halford Mackinder (1861-1947), quien elaboró una recomenda-

1 El concepto de poder blando y poder duro fue acuñado por Joseph Nye de la Universidad de Harvard. El poder blando es la habilidad para atraer y cooptar más que para reprimir. El poder blando es de naturaleza no coercitiva y se manifiesta en la cultura, los valores políticos, las políticas extranjeras y es una forma de proyectar poder de manera sutil y seductora como la propaganda, el marketing político, el cine estadounidense de Hollywood y demás. En tanto, el poder duro es el uso de la fuerza en su carácter violento como la represión o la mutilación que buscan intimidar, reprimir y controlar por medio de la fuerza (Nye 2005).
La geopolítica

de la corrupción en México

Nubia Nieto 
ción estratégica a partir de un "análisis histórico" que relaciona el dominio de una potencia imperial con el entorno físico y el desarrollo tecnológico de los medios de transporte (Mackinder 1904). Las aportaciones del general alemán Karl Haushofer (1869-1946), cuya tesis se funda en la autosuficiencia de los países, es decir, la capacidad de basarse en sus propios recursos sin depender de otros Estados y poder desarrollar la inteligencia militar para lograrlo (Haushofer 2012). Por otra parte, no se puede pasar por alto las contribuciones de la escuela de la geopolítica crítica representada por Danilo Zolo, Peter Taylor y Colin Flint. La perspectiva de la geopolítica crítica expone una pluralidad de prácticas representativas generadas desde los diferentes centros de poder social, cultural, económico, y no como la singularidad que proponen los intelectuales de Estado y los principales actores hegemónicos. Mientras tanto la geopolítica clásica ha estado vinculada y generada desde las estructuras de poder y conocimiento predominantes (González 2017). En este sentido, la geopolítica provee de herramientas teóricas y pragmáticas para el entendimiento del impacto regional, nacional y global de la corrupción, tomando en cuenta que la corrupción rebasa fronteras nacionales, y alcanza dimensiones globales. La geopolítica como disciplina que integra asuntos estratégicos y de seguridad -teniendo en cuenta que la corrupción se ha convertido en un asunto de seguridad nacional para México- adquiere mayor relevancia. Además, si a ello agregamos la posición geográfica de México, como factor clave en el panorama geopolítico mundial, la geopolítica se convierte en una disciplina fundamental para comprender el desarrollo de la criminalidad mundial. Por otra parte, los actores involucrados en asuntos de corrupción operan cada vez más de manera internacional, toda vez que sus operaciones están conectadas a nivel global, mismo si la fuente de extracción de la corrupción se realiza en un país específico, pero acaba en bancos o paraísos fiscales que operan a nivel internacional. La corrupción política se aleja más de patrones locales para mezclarse con otros crímenes como el lavado de dinero, narcotráfico, tráfico de órganos y tráfico de migrantes, entre otros, cuyas redes globales pueden ser entendidas gracias a la ayuda de la geopolítica.

En este sentido, la geopolítica permite dibujar líneas de análisis en el desarrollo de la corrupción en México, debido que es un fenómeno que permea a la estructura del poder que atraviesa no solo una década, ni un siglo, sino ha permeado la estructura del Estado desde su fundación. La complejidad de la corrupción mexicana opera bajo una combinación de factores. Por un lado, México es un punto geográfico clave. Al norte con el país más influyente geopolíticamente a nivel global ${ }^{2}$ y al sur con la región latinoamericana, que

2 Para algunos estudiosos de la geopolítica, América Latina, y México, en particular, es un país clave para entender las políticas estadounidenses a nivel mundial, debido a que ha sido considerado "el laboratorio de los Estados Unidos" por años en materia de políticas internacionales, pues lo que implemente en su vecino país del sur será exportado al resto del mundo. Desde sus políticas expansionistas territoriales que datan del siglo XIX entre la Guerra de Estados UnidosMéxico (1846-1848) y la anexión posterior de Texas (1845), California (1850), Nevada (1864), 
aún arrastra enormes problemas de subdesarrollo económico tales como altos niveles de desigualdad social, conflictos sociales, altas tasas de desempleo, acceso desigual a recursos naturales, de infraestructura pública, de violencia causada por grupos criminales, de educación y salud, entre otros (Nieto 2004). Por otro lado, las representaciones del poder y el funcionamiento de las instituciones políticas en México están permeadas de relaciones de corrupción. La corrupción es usada como una herramienta del poder suave o soft power para influir políticas gubernamentales, orientar presupuestos sociales y de infraestructura pública, determinar cargos administrativos de todos los niveles, manipular el Estado de derecho, manipular elecciones locales, regionales y nacionales, y por supuesto el ejercicio y la proyección del poder político a través de esquemas de socialización basados en la corrupción (Nieto 2011).

\section{La corrupción como un riesgo de la geopolítica global contemporánea}

Actualmente, la corrupción representa una fuente de riesgo geopolítico tanto para los países con democracias consolidadas, democracias recién instaladas, como para los países en transición, o bajo regímenes dictatoriales. El estudio de la corrupción desde el enfoque de la geopolítica es fundamental para entender los riesgos y las amenazas que este fenómeno trae consigo a nivel mundial. En todo el mundo, gobiernos de izquierda, centro y derecha han caído o han sido desestabilizados a causa de imputaciones por corrupción. Instituciones internacionales, organismos no gubernamentales y grupos empresariales tampoco son inmunes a este fenómeno.

La corrupción entendida en un sentido más amplio es utilizada en este texto como "el uso de una función pública para intereses privados, en otras palabras, el uso de una posición oficial, rango o estatus de un funcionario público para su beneficio personal. Ejemplos de conducta corrupta son el soborno, extorción, fraude, malversación de fondos públicos, nepotismo, amiguismo, y apropiación de bienes públicos para uso privado y el tráfico de influencias" (Myint 2000: 35).

La corrupción continúa representando un enorme riesgo geopolítico a nivel mundial toda vez que en varios países la corrupción ha causado una serie de manifestaciones públicas, movilizaciones políticas, incluso movimientos armados para derrocar a sus líderes, aunque cabe señalar que muchas veces también se utiliza a la corrupción como excusa para desacreditar a oponentes

Utah (1896), Arizona (1912), New Mexico (1912), Kansas (1861), Colorado (1876), Wyoming (1890), Oklahoma (1907). Pasando por la exportación de sus modelos económicos, comerciales y de inversión, hasta la reactivación de su economía a través de la industria armamentística, por medio de venta de armas de alto calibre tanto a miembros del ejército como a grupos de narcotraficantes en México (Williams 1957: 3-30, Chomsky 2014: 162).
La geopolítica

de la corrupción en México

Nubia Nieto 
políticos o restar legitimidad a líderes políticos. Un ejemplo de acusaciones de corrupción contra un gobierno latinoamericano lo constituye el caso de Dilma Rousseff, en Brasil, quien asumió el poder el 1 de enero del 2011 y fue suspendida el 12 de mayo del 2016, y remplazada el 31 de agosto del 2016 por Michel Temer, del Partido del Movimiento Democrático Brasileño, quien, a su vez, también fue acusado de recibir sobornos de 152.000 dólares americanos (Minsberg 2016). Años más tarde, el presidente Jair Bolsonaro, en función desde el 1 de enero de 2019, ha sido ya involucrado en asuntos de corrupción que van de nepotismo, clientelismo, tráfico de influencias y evasión fiscal (El País 2019).

En Grecia, la corrupción provocó la caída del sistema financiero, debido a supuestas malversaciones de fondos públicos, evasión de impuestos, manipulación del sistema judicial, nepotismo y demás cargos que llevaron a la Unión Europea y al Fondo Monetario Internacional a implementar el mayor rescate financiero en la historia por 240 billones de euros, cerca de 324 billones de dólares (Smith 2014).

En Reino Unido, la corrupción también ha sido una variable de desestabilización política y de deslegitimación en las instituciones políticas, por ejemplo, el abuso de recursos públicos cometido por miembros del parlamento, quienes solicitaron pagos extraordinarios de más de 700 mil libras (más de un millón 99 mil dólares), por supuestos gastos en el extranjero, hoteles, pago a taxis de hasta 3.300 euros (unos 4 mil 455 dólares), así como la compra de artículos de lujo como una casa para patos de jardín por valor de 1.600 libras, unos 2 mil 512 dólares (Martin 2016). Sin olvidar el tráfico de influencias en el que varios miembros de la Cámara de los Lores ofrecían favores legislativos a cambio de los pagos por tarifas de 111 mil euros anuales, unos 150 mil dólares (Oppenheimer 2010, X-rates 2010). ${ }^{3}$ Son una muestra que ningún país ni institución está inmune a la corrupción.

Entre noviembre del 2010 y junio del 2011, la llamada "primavera árabe" causó inestabilidad política en varios países de Oriente Medio y el Norte de África, notablemente en Egipto, Túnez, Libia, Yemen, Siria y Jordania. Uno de los argumentos que generaron las protestas en la región fueron las acusaciones de corrupción e impunidad contra sus gobiernos (Chulov 2018).

En Estados Unidos, la lista de escándalos de corrupción no deja de aflorar, desde la figura misma del presidente Donald Trump (2017- proyectado 2021), pasando por sus colaboradores más cercanos, hasta miembros del congreso. Por ejemplo, el ex presidente de la campaña de Trump, Paul Manafort, y su socio de negocios, Rick Gates, fueron acusados de conspiración contra Estados Unidos, conspiración por blanqueo de dinero, hacer declaraciones falsas y abuso de recursos públicos (Zapotosky et al. 2017). Otro caso de corrupción en Estados Unidos fue el cometido por el secretario de Salud y Servicios $\mathrm{Hu}-$

3 Las tasas de cambio para hacer las equivalencias indicaban 1 euro = 1,35 dólares, 1 libra=1,57 dólares. Según la tasa de cambio del 31 de diciembre 2010 (X-rates 2010). 
manos, Tom Price (2017-2017), durante la administración de Trump. Price fue obligado a renunciar el 29 de septiembre de 2017 después de que se descubriera que gastó cientos de miles de dólares de los contribuyentes en vuelos privados y lujos personales (Zapotosky et al. 2017). Estados Unidos como país de influencia estratégica a nivel global tampoco se libra de los efectos negativos de la corrupción. De acuerdo con un reporte de Transparencia Internacional (TI 2017), sobre la percepción de las instituciones más corruptas en Estados Unidos, el presidente y sus colaboradores, es decir la Casa Blanca, es percibida como la institución más corrupta en el país, alcanzando el 44\%; seguida de los miembros del congreso que obtienen un $38 \%$. En el tercer lugar se ubican los oficiales del gobierno con un 33\%, y de manera continua, los ejecutivos de negocios 32\%, el gobierno local 23\%, líderes religiosos 22\%, oficiales de impuestos $21 \%$, Policía 20\%, jueces y miembros del magistrado estadounidense $16 \%$ (TI 2017).

En todo el mundo, la corrupción es capaz de reconfigurar fuerzas nacionales e internacionales, movilizar diferentes actores políticos, sociales y civiles, y trazar nuevas líneas en el mapa de la geopolítica global. Según Moran: "En las economías de todo el mundo, la desafección pública causada por el disgusto casi universal de la corrupción y los escándalos financieros está destrozando el status quo de la política global, y el desprestigio de los líderes políticos, por lo cual es urgente analizar el riesgo político que representa la corrupción" (Moran 2016).

En el caso de México, la corrupción se ha convertido en un asunto de seguridad nacional, no sólo porque los actores involucrados son élites políticas de alto nivel, sino también porque este fenómeno involucra otros crímenes que se entrelazan entre sí como el narcotráfico, tráfico de armas y tráfico de seres humanos entre otros. Según el reporte titulado México. Anatomía de la corrupción, el cual fue presentado en 2015 por la organización civil "Mexicanos contra la corrupción y la impunidad”, la corrupción cuesta a México más de 906 mil 358 millones de pesos -alrededor de 44 mil 58 millones de dólares- lo que representa el 5\% del Producto Interno Bruto (PIB). En tanto, la Confederación Patronal de la República Mexicana (Coparmex) considera que la corrupción causa pérdidas anuales de más de un billón 920 mil millones, -alrededor de 93 mil 331 millones de dólares-, equivalente al 10\% del PIB (Redacción 2016: $6)^{4}$. En la misma tónica, la Organización de Estados Americanos (OEA) señala que el costo de la corrupción en México es 5 veces mayor al que se registra a nivel mundial. Según la OEA, las perdidas por actos de corrupción en el mundo equivalen al 2\% del PIB, mientras que en México alcanzan hasta el $10 \%$. Lo que significa que las pérdidas por corrupción a nivel mundial vayan entre 1,5 a 2 billones de dólares (Redacción 2016: 6). En términos de impunidad, la probabilidad de que un delito se denuncie y esclarezca en México
La geopolítica de la corrupción en México

Nubia Nieto

4 Las tasas de cambio para hacer las equivalencias indicaban 1 dólar $=20,5720$ pesos mexicanos. Según la tasa de cambio de octubre 2016 (Tasas de cambio 2016). 
es de 1,14\%, según indican las organizaciones Impunidad Cero y Jurimetría (Arellano 2018: 14). El análisis de la corrupción es cada vez más difícil no solo por la fusión con fenómenos globales, sino también por la impunidad generada al interior de los sistemas políticos nacionales como es el caso de México. De acuerdo con el Índice de Percepción sobre la corrupción 2018, que encuesta a 180 países, dando una nota de 0 a 100, donde 0 representa mayor nivel de corrupción y 100 menor nivel de corrupción, México alcanza el lugar 138 con un índice de 28 sobre 100, lo que representa mayor nivel de corrupción y menor transparencia, colocando a México al mismo nivel que Honduras, Laos y Rusia (TI 2018).

La corrupción representa un riesgo geopolítico para la seguridad nacional del país, sobre todo cuando las élites políticas utilizan el discurso anticorrupción con sentido propagandístico, de diferenciación política con respecto a sus predecesores o de venganza política, más que con sentimientos de compromiso democrático. La presencia de la corrupción a nivel mundial y, particularmente, en México hacen replantear el concepto de democracia, el cual según Schmitter (2007) debe incorporar elementos de rendición de cuentas de los gobernantes no solo al finalizar su gestión o durante las campañas políticas, sino como elemento de evaluación constante durante toda la gestión de los gobernantes ${ }^{5}$.

\section{La corrupción en México: Un riesgo geopolítico latente}

El estudio de la corrupción en México es complejo debido a que frecuentemente está relacionada con otros fenómenos como el narcotráfico, el secuestro, el tráfico de órganos, la trata de personas, el tráfico de especies en vías de extinción, la compra de armas de alto calibre o de uso exclusivo del ejército, el asesinato a periodistas, la manipulación en los medios de comunicación, el otorgamiento de contratos públicos, el lavado de dinero y la operación de negocios con compañías transnacionales. Así pues, la corrupción en México no opera de forma aislada ni es solo un fenómeno local o regional de algunos

\footnotetext{
5 La democracia tiene muchas definiciones, implicaciones y consecuencias, pero la rendición de cuentas es uno de sus principales componentes, por lo cual el enfoque adoptado en este texto pone el énfasis hacia la rendición de cuentas. La participación ciudadana, la igualdad política, la consciencia cívica, el comportamiento decente de las autoridades, el sentido de la eficacia política individual, el respeto por las normas constitucionales, la protección de los derechos humanos, la libertad de prensa, el respeto a la opinión pública, la búsqueda de la reducción en los niveles de desigualdad social y la libertad de asociación son algunos de los componentes que se incluyen en esta noción. Así, la democracia es un régimen o sistema de gobierno en el cual sus gobernantes son llamados a rendir cuentas por sus acciones en el dominio público por los ciudadanos, actuando indirectamente a través de la competencia y la cooperación de sus representantes o instituciones mediatoras, y pueden ser llamados a rendir cuentas en cualquier momento de su gestión, con posibilidad abierta a ser removidos según la ciudadanía lo considere pertinente (Schmitter 2007: 2).
} 
funcionarios corruptos, sino, por el contrario, opera bajo esquemas de cooperación internacional.

La globalización también contribuye a hacer más complejo el estudio de la corrupción. La situación geográfica de México, como país transitorio a los Estados Unidos, mercado altamente cotizado por el crimen organizado, tampoco facilita el entendimiento simple de la corrupción. Un ejemplo coyuntural de la geopolítica de la corrupción que muestra la complexidad de nexos entre corrupción, lavado de dinero, tráfico de influencias, narcotráfico son las revelaciones de los Panama Papers, los cuales conforman 11,5 millones de documentos que involucran a líderes políticos de todo el mundo, miembros de la aristocracia mundial, narcotraficantes, personajes de la farándula y empresarios. Los paraísos fiscales tienen como objetivo la protección de activos contra robos, la confidencialidad de bienes y la evasión fiscal. En la mayoría de los casos han servido para evitar pagar impuestos, de allí la naturaleza "ilegal de estos fondos". Aunque "tener esos recursos en esos países no lo hace ilegal, lo que lo hace ilegal es no pagar impuestos en los países de donde procede la riqueza" (Vanguardia 2016). Entre los mexicanos involucrados con la firma panameña Mossack Fonseca, responsable de gestionar los paraísos fiscales albergados en las Islas Caimán, las Bahamas y las Islas Británicas, está el dueño de la empresa Higa, Juan Armando Hinojosa, quien se vio beneficiado de millonarios contratos del gobierno mexicano, luego de haber vendido una lujosa vivienda por una cantidad simbólica a Angélica Rivera, esposa del presidente Enrique Peña Nieto (2012-2018), y quien ocultó más de 100 millones de dólares en paraísos fiscales en las Islas Nieves y las Islas Vírgenes Británicas. También se menciona al ex director de Petróleos Mexicanos (Pemex), Emilio Lozoya (2012-2016); al contratista de Pemex, Ramiro García; al narcotraficante colombiano Jorge Milton Cifuentes, socio del "Chapo Guzmán" (Joaquín Archivaldo Guzmán Loera, líder del cártel de Sinaloa, capturado en enero del 2016); al prominente empresario y presidente de Televisión Azteca, Ricardo Salinas Pliego ${ }^{6}$ (Vanguardia 2016, Corona 2016). También aparecen: Alfonso de Angoitia, un alto ejecutivo de Televisa, una cadena de televisión mexicana; la finada actriz Edith González, y el dueño de la firma Oceanografía, Amado Yáñez, acusado en 2014 de defraudar al banco estadounidense Citi, y utilizar documentos falsos para obtener créditos bancarios por más de 400 millones de dólares a través de su filial bancaria Banamex - anteriormente conocido como Banco Nacional de México (Vanguardia 2016, Corona 2016).

La complejidad de la corrupción en todo el mundo, y en México en particular, hace de este fenómeno un elemento clave del nuevo entramado geopolítico mundial. Las redes del poder político global se entrelazan con otros crímenes como el lavado de dinero, crimen cibernético, paraísos fiscales y tráfico de

6 De acuerdo con Forbes, la fortuna de Ricardo Salinas Pliego asciende a $\$ 7.3$ billones de dólares, y se ubica en el lugar número 8 de los hombres más ricos de México. Salinas Pliego funda su riqueza en dos compañías: Televisión Azteca y el Grupo Elektra (Forbes 2018).
La geopolítica de la corrupción en México

Nubia Nieto 
drogas, entre otros. Además, los actores involucrados son de todo el mundo, sus riquezas pueden cruzar fronteras y desestabilizar gobiernos, alterar tasas de cambio, desquebrajar sistemas económicos y provocar crisis en los mercados financieros. No es casual que incluso bancos de talla mundial como el banco Hong Kong and Shanghai Banking Corporation (HSBC) se vean involucrados en "transacciones turbias" que atraviesan a todos los continentes. Por ejemplo, entre 2006-2010, “el Cartel de Sinaloa utilizó al banco HSBC para lavar 881 millones de dólares producto del negocio de la droga. HSBC México aceptó cientos de miles de dólares en efectivo en una sola cuenta, incluso utilizaba cajas diseñadas especialmente para ajustar a las cantidades a depositar" (Wainwright 2016). Aunque HSBC fue multado con 1,9 millones de dólares por lavado de dinero proveniente del narcotráfico, este banco se ha visto nuevamente involucrado en otros casos de corrupción, y se le ha vuelto a multar con 353 millones de dólares por evasión de impuestos de clientes franceses en noviembre de 2017; otra multa en la misma fecha por 51 millones de dólares por fallas con el banco privado suizo; otra multa adicional en septiembre del 2017 por 175 millones de dólares debido a prácticas ilegales en manipulación de divisas, y una más en enero de 2018 por 101,5 millones de dólares nuevamente por manipulación de divisas (English 2018).

La mayor parte de escándalos de corrupción política en México está directamente vinculada a otros fenómenos globales de igual complejidad, en los cuales también se ven involucrados firmas o grupos que operan a nivel internacional, como hemos ejemplificado anteriormente de manera general con el banco HSBC. A nivel nacional, gobernadores de diferentes estados de la República Mexicana han sido involucrados no sólo en asuntos de corrupción política tales como tráfico de influencias, nepotismo, abuso del cargo público para beneficio privado, sino también en lavado de dinero, nexos con el narcotráfico, relaciones con redes de pederastas, tráfico de migrantes, y tráfico de armas de alto calibre entre otros crímenes, que atraviesan fronteras internacionales. Un caso por citar es el ex gobernador del Estado de Veracruz, Javier Duarte de Ochoa (2010-2016), acusado de desviar 73 mil millones de pesos provenientes de recursos públicos, unos 3.800 millones de dólares. Esto quiere decir que en la administración de Duarte desaparecían, en promedio, 28 mil pesos de las arcas públicas por minuto (1.495 dólares) (Ángel 2017). Duarte Ochoa fue detenido en julio del 2017 y condenado a sólo nueve años de cárcel y con la posibilidad de reducir su condena a la mitad, luego de haberse reconocido culpable de lavado de dinero y asociación delictuosa. Los cargos de corrupción en su contra tales como peculado, tráfico de influencias, ejercicio indebido del cargo público y robo no fueron imputados como delitos graves (García, Ferri 2018), y lo que es peor la investigación en su contra cubría solo nexos a nivel estatal, sin indagar sus nexos a nivel nacional e internacional, a pesar de que ese tipo de operaciones delictivas no operan a escondidas ni del gobierno nacional ni internacional. Además, los colaboradores cercanos de Duarte Ochoa como Arturo Bermúdez, jefe de la policía durante su gobierno, 
acusado entre otros delitos de la desaparición forzada de 15 personas en el estado de Veracruz, fue encarcelado solo por unos meses y puesto en libertad. Lo mismo ocurrió con Pacho Viejo y Mauricio Audirac, extesoreros, Francisco Valencia, exsecretario de Obras Públicas, y Juan Antonio Nemi Debi, exsecretario particular de Duarte, todos acusados de delitos como peculado o tráfico de influencias (García, Ferri 2018). Duarte Ochoa es uno de varios gobernadores involucrados en asuntos de corrupción y crimen organizado en México, sin embargo, la pregunta más inquietante es, ¿cómo logra un gobernador de un Estado acumular tanto dinero y poder, sin ser notado por las autoridades centrales?, ¿cómo se entretejen las relaciones de poder y corrupción entre otros miembros de la élite política nacional e internacional para poder llevar a cabo transacciones monetarias complejas? Las repuestas no son simples, sin embargo, lo que salta a la vista, es que tales niveles de corrupción e impunidad no pueden ser llevadas a cabo por un solo hombre ni una sola entidad, sino por complejas redes del poder nacional en el que diputados locales, regionales, nacionales, miembros de la policía, jueces y demás miembros del poder judicial, federal y ejecutivo se protegen mutuamente para violentar el Estado de derecho con tanta facilidad, sin olvidar las redes internacionales del crimen organizado que apoya sus operaciones.

En África, como en América Latina, se observa una correlación entre corrupción y vulnerabilidad del Estado de derecho. En el caso de México esta correlación se hace evidente toda vez que los altos niveles de corrupción han facilitado la vulnerabilidad del Estado de derecho, proporcionando tierra fértil para el desarrollo del crimen organizado y el aumento en la violencia (Astorga 1999). Por ejemplo, tan solo en 2017 (de enero a octubre) se registraron 20.878 homicidios reportados oficialmente, más los homicidios que se quedan sin reportar, cuerpos no identificados o cuerpos desaparecidos. Lo que significa que cada 16 minutos se registra una víctima por homicidio. También se registraron aumentos en la extorsión, robo con violencia, robo a negocios, robo a casa habitación, robo de vehículo y robo a transeúntes (Meléndez 2017b). En la mayoría de los casos, las organizaciones delictivas cuentan con la protección de funcionarios públicos, policías y miembros del ejército a nivel local, estatal y federal, lo que les facilita violentar el Estado de derecho (Astorga 1999). La corrupción en México ha facilitado el desarrollo del crimen organizado, multiplicando los riesgos geopolíticos mundiales. El gobierno mexicano reconoce más de 200 células criminales que operan en el país. Además, se reportan entre 80 mil y 100 mil personas asesinadas desde 2006 hasta mediados del 2018 (CFR 2018), más los asesinados no registrados oficialmente, debido que han sido reportados como desaparecidos o incluso han pasado desapercibidos por las autoridades. El crimen organizado ha causado también el desplazamiento de más de un cuarto de millón de personas (Grillo 2013).

Por otro lado, los altos niveles de corrupción también repercuten en la recomposición del mapa geopolítico de la libertad de prensa mundial. Se trata, particularmente, de los ataques a periodistas -infringidos por siste-
La geopolítica de la corrupción en México

Nubia Nieto 
mas autoritarios, crimen organizado y funcionarios corruptos- que cada vez están más presentes en varios países, especialmente en México. De acuerdo con Reporteros sin Fronteras (RFS), México es un país de alto riesgo para ejercer el periodismo, alcanzando en 2018 el lugar 147 de una lista de 180 países, con una nota de 48.91 en una escala de 0 a 100, donde cero representa mayor libertad de prensa y menor riesgo para ejercer el periodismo, y 100 indica exactamente lo contrario, es decir, el peor lugar para ejercer la libertad de expresión. En este contexto, ejercer el periodismo en México es más peligroso que hacerlo en Pakistán que ocupa el lugar 139, con una nota de 43.24; Palestina se ubica en el sitio 134 con una nota de 42.96; Kuwait que se ubica en el lugar 105 y tiene nota 31.91; o Afganistán que se coloca en el sitio 118, y obtiene una nota de 37.28 (RSF 2018). De acuerdo con RFS, en México, cuando los periodistas cubren noticias de corrupción relacionadas con funcionarios mexicanos son confrontados al dilema "plata o plomo", aceptan dinero por su silencio o firman su sentencia de muerte. Además, según RFS, la tendencia es cada vez mayor al asesinato de periodistas sin darles la opción de comprar su silencio.

\section{Propuesta de lucha contra la corrupción}

Técnicamente México cuenta con procesos y procedimientos electorales propios de las democracias occidentales, los ciudadanos tienen la capacidad de elegir a su gobernantes, locales, regionales y nacionales, sin embargo, hasta antes del 2018, una vez que los representantes políticos eran elegidos, obtenían inmunidad política y jurídica para actuar de manera caprichosa, personalista o clientelista. La corrupción en México no solo ha representado un asunto de cifras y déficit en las cuentas públicas, sino también el deterioro de la calidad de vida de millones de habitantes, sobre todo de sectores más vulnerables, exclusión social, debilitamiento de las instituciones públicas, incredulidad en la ética pública y política, reproducción de esquemas de poder colonialistas basado en relaciones personales tales como el nepotismo, amiguismo, compadrazgo y clientelismo, entre otros.

Cabe destacar que luego de las elecciones celebradas el 2 de julio del 2018 en México, el nuevo presidente electo Andrés Manuel López Obrador (AMLO), de la coalición "Juntos haremos historia" compuesta por el Partido de los Trabajadores (PT), Movimiento de Regeneración Nacional (Morena) y Encuentro Social, en respuesta a sus compromisos de la campaña política, ha propuesto 50 lineamientos para el combate a la corrupción pública entre los que destacan:

1. Reforma del Artículo 108 de la Constitución para que el presidente en funciones pueda ser juzgado por delitos electorales y relacionados con actos de corrupción.

2. Suspensión de todos los fueros y privilegios para funcionarios públicos. 
3. Reforma de la ley para considerar como delitos graves el tráfico de influencias, la corrupción, la asociación entre funcionarios y particulares para cometer fraudes a la cuenta pública, el robo de combustibles y fraude electoral en cualquiera de sus modalidades. Las penas no permitirán al inculpado la obtención de la libertad bajo fianza.

4. La fiscalía general contará con absoluta autonomía, no recibirá consigna alguna de la presidencia y sus prácticas apelarán al principio del derecho liberal al margen de la ley, nada y nadie por encima de la ley. (Milenio 2018) Los 50 lineamientos se dirigen directamente a la reducción de privilegios, favoritismos, nepotismo y abuso de recursos públicos por parte de los burócratas, por lo cual abren una nueva esperanza en la gestión del poder político en México y en la reducción de la corrupción e impunidad. No obstante, seis años de gobierno es ciertamente un periodo corto para sanar siglos de atraso, desigualdad social, corrupción, nepotismo y esquemas autoritarios y colonialistas en el ejercicio del poder político.

Por otro lado, la rendición de cuentas en cualquier gobierno es un punto central para el funcionamiento de los regímenes políticos. Según Schmitter (2007), cuanto mayor es el nivel de rendición de cuentas en un gobierno, más democrático es un régimen y mejor calidad de gestión y legitimidad alcanzan sus gobernantes, además de contribuir a reducir los espacios para la corrupción. Hasta ahora la corrupción política ha sido asociada principalmente a gobiernos autoritarios, en transición o con bajos niveles de respeto a los derechos humanos, sin embargo, lo cierto es que la corrupción es un fenómeno mundial que florece tanto en democracias consolidadas, países altamente industrializados como en países en vías de desarrollo, por lo cual "la corrupción representa una fuente de riesgo geopolítico global" (Moran 2016). De acuerdo con Moran, "la lista de gobiernos que han caído, han sido amenazados o han sido destituidos, debido a la corrupción es larga y cada vez tiende en crecer. Desde la crisis financiera de 2008, puede observarse un punto en común en varios países que son los altos niveles de corrupción" (Moran 2016).

Ciertamente, hay una lista enorme sobre acuerdos internacionales que condenan y sancionan a la corrupción. Sin embargo, lo que este fenómeno ha demostrado también es que cada vez es más difícil separarlo de otros problemas globales, en los cuales todos los gobiernos tienen que asumir su responsabilidad si realmente quieren combatir a la corrupción que atraviesa fronteras y es capaz de reconfigurar los esquemas geopolíticos contemporáneos. En este contexto, gobernantes de todo el mundo, y de Estados Unidos en particular ${ }^{7}$,

7 El desarrollo de la corrupción en varios países está ampliamente ligado a políticas permisivas estadounidenses, pues no es una casualidad que la mayoría de los dictadores, presidentes y funcionarios a nivel mundial han podido ejercer esquemas de corrupción sistémica basados en nepotismo, clientelismo, amiguismo, enriquecimiento ilícito, impunidad, sin olvidar violación de derechos humanos con tanta facilidad. En la mayoría de casos todos han prosperado al amparo estadounidense, por ejemplo: Nicolae Ceaușescu, presidente de Rumania (1974-1989), Haji Suharto, presidente de Indonesia (1967-1998); Ferdinand Marcos, presidente de Filipinas

\section{La geopolítica}

de la corrupción en México

Nubia Nieto 
tienen que abandonar el doble discurso contra la corrupción, el cual condena esta práctica en papel, pero se permite en tanto se ajuste a los intereses de grupos de poder ${ }^{8}$. México es un ejemplo de los costos humanos que engendra la corrupción cuando se entrelaza con el narcotráfico. Desde luego, el desarrollo del narcotráfico en México no sería posible sin el amparo de autoridades nacionales e internacionales, pues dicho fenómeno no florecería con tanta facilidad sin la protección de los gobiernos ${ }^{9}$.

\section{Comentarios finales}

El presente análisis intenta responder a las preguntas iniciales, arrojando algunas observaciones generales.

En el primer lugar, la corrupción a nivel internacional representa un nuevo riesgo geopolítico global que amenaza tanto a las democracias consolidadas como a las democracias jóvenes o en proceso de nacimiento, y a todos los sistemas políticos contemporáneos. La corrupción también amenaza a las economías desarrolladas y a las emergentes como se ha podido observar en los casos descritos en los Panama Papers y en los escándalos de corrupción en México.

En el segundo lugar, las características de la corrupción en México son cada vez más difíciles de distinguir toda vez que el fenómeno de la corrupción

(1965-1986), Jean-Claude Duvalier, presidente de Haití (1971-1986); Chun Doo-hwan, presidente del Sur de Corea (1979-1988), Manuel Noriega Moreno, presidente de Panamá (19831989), Mobutu Sese Seko, presidente de la República Democrática del Congo (Zaire) (19651997), Alberto Fujimori en Perú (1990-2000), entre otros (Chomsky 2014; Becker, Sklar 1999).

8 Según Dale, el desarrollo del narcotráfico está íntimamente ligado a la protección de las agencias de inteligencia, particularmente a la Agencia Central de Inteligencia (CIA - Central Intelligence Agency): "Los investigadores están cada vez más conscientes de un tercer aspecto en el desarrollo del narcotráfico: la protección de las agencias de inteligencia y las conexiones con la droga. Dentro de la burocracia del gobierno estadounidense, las agencias de investigación y de las fuerzas especiales han explotado recurrentemente a los narcotraficantes y a sus aliados políticos corruptos para sus propios intereses en operaciones antisubversivas o anticomunistas" (Dale 2000: 2-4). Por ejemplo, dice Dale, "en México, el aliado más cercano de la CIA por años fue la Dirección Federal de Seguridad, (...) cuyo jefe era Miguel Nasser Haro (1978-1982), quien era un agente de la CIA y entregaba credenciales de alto nivel a traficantes mexicanos, tales permisos eran autorizados por la DEA (Drug Enforcement Administration) - administración para el control de drogas, como 'licencias para traficar' (...) El Cartel de Guadalajara, el cartel más poderoso en los años 80 , prosperó gracias a la protección de Nasser Haro, una adquisición de la CIA" (Dale 2000: 3-4).

9 En México solo, decenas de miles de personas han sido asesinadas relacionadas con el tráfico de drogas. Y Estados Unidos es la fuente del problema, en doble sentido, actualmente -en términos de demanda, la cual es obvia, y también como suministro, la cual es poco discutida. Las armas de los cárteles mexicanos vienen de los Estados Unidos. La Oficina de Alcohol, Tabaco y Armas de Fuego, una oficina del gobierno federal, analizó la procedencia de las armas confiscadas en México. De acuerdo con sus datos, cerca del 70\% procedían de los Estados Unidos (Chomsky 2014: 162). 
se funde con otros crímenes de igual complejidad como el narcotráfico, el lavado de dinero o el tráfico de armas que operan a nivel internacional. Ciertamente, la corrupción no es lo mismo que el crimen organizado, sin embargo, este último aprovecha las zonas grises que genera el clientelismo, el amiguismo, el parentesco o el tráfico de influencias para reforzar su alcance.

En el tercer lugar, entre los factores más importantes que han provocado que la corrupción en México amenace la estructura del Estado son, a nivel nacional, el abuso del poder, el ejercicio del nepotismo, el clientelismo, la impunidad, la desigualdad social, la descomposición del poder político, la falta de transparencia y rendición de cuentas con la ciudadanía y, desde luego, la presencia del crimen organizado. A nivel internacional, el rol de corporaciones bancarias y financieras globales que favorecen la corrupción mexicana y el lavado de dinero, el doble discurso de gobiernos desarrollados, particularmente del gobierno estadounidense, para culpabilizar y estigmatizar a México como generador de violencia, narcotráfico y corrupción, cuando en realidad es una responsabilidad compartida tanto por las instituciones globales como nacionales. No es casual que el dinero fruto de la corrupción mexicana acaba en las arcas de los bancos estadounidenses, británicos, suizos, españoles o de Luxemburgo, entre los más destacados. Tampoco es casual que muchas compañías globales, particularmente fabricantes de armamento se ven beneficiadas de la violencia que impera en México. Sobre todo, si se considera las ganancias millonarias que obtienen por la venta de armamento, cuyos clientes son tanto el ejército mexicano como miembros del crimen organizado. De acuerdo con la Oficina de Washington para América Latina (WOLA) en su informe titulado Cuatro hechos sobre la legislación de armas y la violencia de los carteles en México indica:

Los carteles mexicanos de la droga obtienen la mayoría de sus armas de los Estados Unidos. El 70\% de las armas recuperadas en México y enviadas para su rastreo entre 2007 y 2011 provinieron de los Estados Unidos. Las razones son obvias: es casi imposible comprar legalmente un arma en México, pero hay más de 8,000 traficantes de armas en los estados fronterizos de los Estados Unidos. Los carteles mexicanos de la droga pueden adquirir armas fácilmente en los Estados Unidos a través de compradores de paja o en exhibiciones de armas. (WOLA 2013)

En México, las cifras de muertes relacionadas al tráfico de drogas alcanzan o superan a las de una guerra civil. Mientras que el número de muertes a civiles en Afganistán alcanzaron más de 26.000 desde 2001 hasta 2014, y en Irak de 160.500 desde 2003 a 2014, en México el número de homicidios relacionados al narcotráfico alcanzó 164.000 entre 2007-2014. Incluso en el mismo periodo (2007-2014) el número de muertes en Irak y Afganistán fue de aproximadamente 103.000 (Breslow 2015). Lo que demuestra que los costos humanos son más altos en México, bajo una guerra civil silenciosa causada
La geopolítica

de la corrupción en México

Nubia Nieto 
por el crimen organizado, que en Irak o Afganistán bajo guerras civiles e intervenciones extranjeras.

El tema del crimen organizado en México da material para ser estudiado por separado, por lo cual solo se hace una breve mención en tanto que la corrupción de funcionarios públicos y políticos facilitaron el desarrollo de varios tipos del crimen organizado tales como el narcotráfico, la venta de armas y las redes de tráfico de personas (Nieto 2011). Otro caso de corrupción en México, que entrelaza la corrupción de funcionarios públicos de la compañía estatal Petróleos Mexicanos (PEMEX) y grupos de criminales en varios estados del país, es lo que es llamado "huachicoleo" -robo de combustible o gasolina- de las tuberías de PEMEX. De acuerdo con un reporte de Forbes, en 2018 se contabilizaron 14.894 tomas clandestinas, lo que representó 147 mil millones de pesos en los últimos tres años a la petrolera mexicana (Solís 2019). La historia del "huachicoleo" se remonta al año 2000, bajo el gobierno de Vicente Fox (2000-2006), cobró fuerza con Felipe Calderón (2006-2012) y llegó a su punto máximo con Enrique Peña Nieto (2012-2018). El 80\% de las tomas clandestinas, desde el año 2016 al 2019, se han concentrado en siete estados: Hidalgo (registrando 2.121 tomas), Puebla (2.072), Guanajuato (1.919), Jalisco (1.550), Veracruz (1.539), además del estado de México (sin registro oficial) y Tamaulipas (sin registro oficial). El crecimiento de tomas clandestinas petrolíferas no pudo darse de manera aislada ni sin el conocimiento de autoridades estatales, locales y nacionales (Solís 2019).

En el cuarto lugar, en las elecciones del 2 de julio 2018, el pueblo mexicano eligió como presidente a AMLO y con ello su propuesta de control del crimen organizado ${ }^{10}$, reducción de violencia y disminución de la corrupción política. Ciertamente, la propuesta de AMLO presenta un enfoque innovador, pero no se debe olvidar que en México la corrupción es compleja y con raíces profundas en la historia del país. La corrupción en México, ciertamente, tiene una larga historia que se remonta a la época colonial (Nieto 2014), sin embargo, este fenómeno se ha visto agravado por la globalización y el crimen organizado mundial, que amplifican su impacto y consecuencias, tales como el narcotráfico, el tráfico de armas, el tráfico humano y el crimen cibernético, entre otros.

En el análisis del desarrollo de la corrupción contemporánea en México no pueden perderse de vista factores geopolíticos tales como la posición geográfica de México y la relación con su vecino del norte ${ }^{11}$, la cual contribuye

${ }^{10}$ El plan de pacificación de AMLO se compone de 5 puntos clave: 1 . Amnistía y desarme de carteles. 2. Legalizar drogas. 3. La policía y fuerzas militares, naval y federal cumplirán tareas de Guardia Nacional. 4. Combate a la corrupción. 5. Combatir impunidad, esclarecer desaparecidos y respetar los derechos humanos (Najar 2018).

${ }_{11}$ Para varios estudiosos, Estados Unidos ha entrado en una crisis geopolítica desde 1970, que pone en riesgo su predominancia mundial y su estabilidad económica, por lo cual, en un intento por reactivar su hegemonía y su economía, ha activado el sector balístico, sector clave para su economía, en varios países entre ellos México, cuyos beneficios por la venta de armas 
a intensificar las consecuencias de otros riesgos geopolíticos globales: tráfico de armas, tráfico de personas y tráfico de drogas, entre otros. Finalmente, el presente trabajo, siendo congruente con su hipótesis inicial, explora el desarrollo de la corrupción a la luz de los riesgos globales, tomando como estudio de caso el desenvolvimiento de la corrupción en México y sus nexos con la corrupción global. El texto arroja como comentario final que el estudio de la corrupción en México es cada vez más complejo debido a la fusión de este fenómeno con otros crímenes globales.

\section{Referencias bibliográficas}

Al-Rodhan N. (2009), Neo-Statecraft and Meta-Geopolitics: Reconciliation of Power, Interests and Justice in the 21st Century, Lit Verlag, London.

Ángel A. (2017), Del Nuevo PRI a la corrupción de Javier Duarte, "The New York Times", 8 de mayo.

Arellano García C. (2018), De 1.14\%, la probabilidad de que un delito se denuncie y esclarezca: análisis, "La Jornada", 17 de julio, p. 14.

Astorga L. (1999), Drug trafficking in Mexico: A First General Assessment, United Nations Educational Scientific and Cultural Organization (UNESCO), France.

Becker D. G., Sklar R. L. (1999), Postimperialism and World Politics, Greenwood Publishing Group, United States.

Breslow J. (2015), The Staggering Death Toll of Mexico's Drug War, "Frontline", 27.07.2015, https://www.pbs.org/wgbh/frontline/article/the-staggering-death-toll-of-mexicos-drugwar/, fecha de consulta: 17.01.2019.

CFR (2018), Criminal Violence in Mexico. Global Conflict Tracker, Council Foreign Relations, New York.

Chomsky N. (2014), Power systems, London.

Chomsky N. (2017), Global Discontents. Conversations on the Rising Threats to Democracy, Hamish Hamilton/Penguin, United Kingdom.

Chulov M. (2018), Hard-Won Victories of Arab Spring Appear More Fragile than Ever, "The Guardian", January 12.

superan los billones de dólares anuales (Chomsky 2014: 70, Stone 2012, Chomsky 2017). Toda vez que Estados Unidos es el mayor productor de armas a nivel mundial, tan solo en 2015 la industria balística generó 49,3 billones de dólares, creó 21.000 nuevos empleos y contribuyó con 6,2 billones de dólares al gobierno estadounidense en impuestos. Y es el único sector que ha crecido exponencialmente desde 1986. La producción masiva de armas en Estados Unidos está destinada tanto al consumo interno como a la venta internacional. Sin contar que es el único sector al que EE. UU. destina mayores recursos. En 2015, el presupuesto del gobierno de EE. UU. para el sector de la defensa fue de 597 billones de dólares, mayor que China, Arabia Saudita, Reino Unido, Rusia, India, Francia, Japón, Alemania, Corea del Sur, Brasil y Australia. Entre las empresas de armas estadounidenses más poderosas están Lockheed Martin (US), Boeing (US), Raytheon (US) más toda la industria de inteligencia, de tecnología y de comercio de armas que desarrolla el sector (Fernandes 2017).
La geopolítica de la corrupción en México

Nubia Nieto 
Corona S. (2016), Los papeles de Panamá. La agencia tributaria de México investigará a los señalados por los papeles de Panamá, "El País", 5 de abril.

Dale Scott P. (2000), Washington and the Politics of Drugs, "Variant", vol. 2, no 11, summer, pp. 2-5. Dodds K. (2014), Geopolitics, 2nd ed., Oxford University Press, United Kingdom.

El País (2019), MP vê indícios de que Flávio Bolsonaro lavou dinheiro ao comprar 19 imóveis por 9 milhões, Editorial El País, São Paulo, Brasil, Mai 16, https://brasil.elpais.com/ brasil/2019/05/16/politica/1557961576_034022.html, fecha de consulta: 17.06. 2019

English S. (2018), HSBC Slapped with \$100m Fine after US Probe, "Evening Standard", January 19, p. 49.

Fernandes Silva J. (2017), The Economics of Firearms, “The market mogul”, 26.02.2017, https://themarketmogul.com/the-economics-of-firearms/a , fecha de consulta: 20.01.2018.

Forbes (2018), Lists of billionaires. Ricardo Salinas Pliego \& family, "Forbes", 11.01.2018, https://www.forbes.com/profile/ricardo-salinas-pliego/, fecha de consulta: 11.01.2018.

García J., Ferri P. (2018), La liberación de la cúpula de Duarte evidencia las carencias de la lucha anticorrupción en México, "El País", 6 de diciembre.

González Tule L. (2017), Organización del espacio global en la geopolítica "clásica": una mirada desde la geopolítica crítica, "Revista de Relaciones Internacionales, Estrategia y Seguridad", vol. 13, no 1, Universidad Militar Nueva Granada, Colombia.

Grillo I. (2013), Mexican Cartels: A Century of Defying US, “The Brown Journal of World Affairs", vol. XX, no 1, winter, pp. 253-265.

Haushofer K. (2012), Los fundamentos geográficos de la política exterior, "Geopolítica(s). Revista de Estudios sobre Espacio y Poder", vol. 3, no 2, pp. 329-336.

Kjellen R. (1916), Staten somlifsforme, Hugo Gebers-Förlag, Stockholm.

Lacoste Y. (1993), Dictionnaire géopolitique, Flammarion, Paris.

Lefèvre H. (2000), La production de l'espace, 4 ed., Ethno-sociologie, Paris.

Lorot P., Thual F. (2002), La géopolitique, $2^{\circ}$ ed., Montchrestien, Paris.

Mackinder H. (1904), The geographical pivot of history, “The Geographical Journal”, vol. 23, no 4, pp. 421-437.

Mahan A. (2013), Análisis de los elementos del poder naval, "Geopolítica”, vol. 4, no 2, pp. 305-334.

Martin W. (2016), After the duck house...where MP's expenses went next, "The Guardian”, May 17.

Meléndez R. (2017a), 2017 el más violento en 20 años, "Unomásuno", 22 de noviembre, pp. 8-9.

Meléndez R. (2017b), 2017, año de pesadilla, “Unomásuno”, 29 de noviembre, pp. 8-9.

Milenio (2018), Los 50 lineamientos de AMLO para la austeridad y contra la corrupción, “Milenio", 15 de julio.

Minsberg T. (2016), Brazil's Impeachment Trial of Dilma Rousseff: Readers React, “The New York Times", May 12.

Moran M. (2016), The Geopolitics of Corruption, "Foreign Affairs", 14.04.2016, https://www. foreignaffairs.com/articles/2016-04-14/geopolitics-corruption, fecha de consulta: 20.01.2018. 
Myint U. (2000), Corruption: Causes, Consequences and Cures, "Asia-Pacific Development Journal", vol. 7, no 2, December, pp. 33-58.

Najar A. (2018), Presidencia de AMLO en México: 5 puntos clave del Plan Nacional de Paz y Seguridad de López Obrador para combatir la violencia y pacificación, "BBC News Mundo", 15.11.2018. https://www.bbc.com/mundo/noticias-america-latina-46217695, fecha de consulta: 30.12 .2018 .

Nieto N. (2004), La géopolitique de la corruption: Le Mexique, une étude de cas, Universidad de la Sorbona, Paris I, Tesis para obtener el grado de doctor en Geopolítica. Tomo l y Tomo 1l, Paris.

Nieto N. (2011), La socialización de las élites políticas mexicanas a través de la corrupción, "Análisis Político", no 71, enero-abril, Instituto de Estudios Políticos y Relaciones Internacionales (IEPRI), Universidad Nacional de Colombia, pp. 165-182.

Nieto N. (2014), Corruption in Mexico: A Historical Legacy, "Social Science Diliman", vol. 10, no 1, January-June, University of the Philippines, pp. 101-116.

Nye J. (2005), Soft Power: The Means to Success in World Politics, Public Affairs, New York.

Oppenheimer W. (2010), Los casos de corrupción enlodando el Reino Unido, "El País", 28 de marzo.

RAE (2015), Diccionario de la Lengua Española, 23a ed., vol. 1., Real Academia de la Lengua Española (RAE), España.

Redacción (2016), Equivale la exacción a 10\% del PIB, “Unomásuno", 27 de octubre, p. 6.

Rood T. (2010), Herodotus' Proem: Space, Time, and the Origins of International Relations, "APIA $\triangle \mathrm{NH}$ ", no 16, pp. 43-74.

RSF (2018), World Press Freedom Index 2017, Reporteros sin Fronteras (RSF), 10.01.2018. https://www.rsf-es.org/grandes-citas/clasificacion-por-paises/, fecha de consulta: 15.11 .2018 .

Schmitter P. (2007), Accountability in 'Real-Existing' Democracies: Meaning and Mechanisms, European University Institute, Firenze.

Smith H. (2014), Corruption still alive and well in post-bailout Greece, "The Guardian", December 3.

Solís A. (2019), Los 5 estados con más huachicoleo durante 2018, "Forbes-México", 23 de enero, https://www.forbes.com.mx/los-5-estados-con-mas-huachicoleo-durante-2018/, fecha de consulta: 16.08.2019.

Soppelsa J., Battesti M., Romer J. C. (1988), Lexique de géopolitique, Dalloz, Paris.

Stone O., Kuznick P. (2012), The Untold History of the United States, Ebury Press, United States.

Tasas de cambio (USD/MXN) (2016), Tasas de cambio históricas de dólares de Estados Unidos a Pesos Mexicanos, "Investing.com", 15.01.2016, disponible en: https://es.investing. com/currencies/usd-mxn-converter, fecha de consulta: 11.11.2018.

Transparency International (2017), How corrupt are different US institutions and groups in Society? USA 2017, 03.11.2017, disponible en: https://www.transparency.org/news/feature/ corruption_in_the_usa_the_difference_a_year_makes, fecha de consulta: 10.01.2018.

Transparency International (2018), Corruption Perception Index 2018: Global Scores, 21.02.2018, disponible en: https://www.transparency.org/news/feature/corruption_ perceptions_index_2017, fecha de consulta: 20.11.2018.
La geopolítica

de la corrupción en México

Nubia Nieto 
Vanguardia (2016), Los mexicanos que aparecen en "Panama Papers", "Vanguardia", 4.04.2016, https://www.vanguardia.com.mx/articulo/los-mexicanos-que-aparecen-en-panama-papers, fecha de consulta: 9.01.2018.

Wainwright, T. (2016), NarcoNomics. How to Run a Drug Cartel, Penguin House, United Kingdom.

Williams Applema W. (1957), Latin America: Laboratory of American Foreign Policy in the Nineteen-Twenties, "Inter-American Economic Affairs", no 11, vol. 2, pp. 3-30.

Williams Applema W., Bacevich A., Gardner L. (2009), The Tragedy of American Diplomacy, $15^{\mathrm{a}}$ ed., W.W. Norton \& Company, London.

WOLA (2013), Four Facts about Gun Legislation and Cartel Violence in Mexico, Washington Office on Latin America (WOLA), Washington.

X-rates (2010), Historical Exchange Rates, 31.12. 2010, disponible en: http://www.X-rates. $\mathrm{com} /$ historical/?from=EUR\&amount=1\&date=2010-12-31, fecha de consulta: 15.01.2018.

Zapotosky M., Helderman R., Leonnig C., Hsu S. (2017), Three Former Trump Campaign Officials Charged by Special Counsel, "The Washington Post", October 30. 